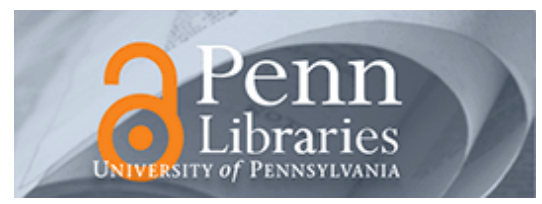

University of Pennsylvania ScholarlyCommons

10-6-2009

\title{
Pressure-temperature phase diagram for shapes of vesicles: A coarse-grained molecular dynamics study
}

Ping Liu

Institute of High Performance Computing

Ju Li

University of Pennsylvania, liju@seas.upenn.edu

Yong-wei Zhang

National University of Singapore

Follow this and additional works at: https://repository.upenn.edu/mse_papers

\section{Recommended Citation}

Liu, P., Li, J., \& Zhang, Y. (2009). Pressure-temperature phase diagram for shapes of vesicles: A coarsegrained molecular dynamics study. Retrieved from https://repository.upenn.edu/mse_papers/172

Copyright 2009 American Institute of Physics. This article may be downloaded for personal use only. Any other use requires prior permission of the author and the American Institute of Physics. Reprinted in:

Pressure-temperature phase diagram for shapes of vesicles: A coarse-grained molecular dynamics study Ping Liu, Ju Li, and Yong-Wei Zhang, Appl. Phys. Lett. 95, 143104 (2009), DOI:10.1063/1.3245307 Publisher URL:

http://link.aip.org/link/?APPLAB/95/143104/1

This paper is posted at ScholarlyCommons. https://repository.upenn.edu/mse_papers/172

For more information, please contact repository@pobox.upenn.edu. 


\title{
Pressure-temperature phase diagram for shapes of vesicles: A coarse-grained molecular dynamics study
}

\author{
Abstract \\ Coarse-grained molecular dynamics simulations are performed to obtain the phase diagram for shapes of \\ a vesicle with a variation in temperature and pressure difference across the membrane. Various \\ interesting vesicle shapes are found, in particular, a series of shape transformations are observed for a \\ vesicle with an initial spherical shape, which changes to a prolate shape, then an oblate shape, and then a \\ stomatocyte shape, with either increasing temperature or decreasing pressure difference across the \\ membrane.

\section{Keywords} \\ biomechanics, biomembranes, biothermics, cellular biophysics, molecular dynamics method, phase \\ diagrams

\section{Comments} \\ Copyright 2009 American Institute of Physics. This article may be downloaded for personal use only. Any \\ other use requires prior permission of the author and the American Institute of Physics. Reprinted in: \\ Pressure-temperature phase diagram for shapes of vesicles: A coarse-grained molecular dynamics study \\ Ping Liu, Ju Li, and Yong-Wei Zhang, Appl. Phys. Lett. 95, 143104 (2009), DOI:10.1063/1.3245307 \\ Publisher URL: http://link.aip.org/link/?APPLAB/95/143104/1
}




\title{
Pressure-temperature phase diagram for shapes of vesicles: A coarse-grained molecular dynamics study
}

\author{
Ping Liu, ${ }^{1} \mathrm{Ju} \mathrm{Li}{ }^{2}$ and Yong-Wei Zhang ${ }^{3, a)}$ \\ ${ }^{1}$ Institute of High Performance Computing, Singapore 117528, Singapore \\ ${ }^{2}$ Department of Materials Science and Engineering, School of Engineering and Applied Science, \\ University of Pennsylvania, Pennsylvania 19104, USA \\ ${ }^{3}$ Department of Materials Science and Engineering, National University of Singapore, Singapore 119260, \\ Singapore and Institute of High Performance Computing, Singapore 117528, Singapore
}

(Received 4 June 2009; accepted 18 September 2009; published online 6 October 2009)

Coarse-grained molecular dynamics simulations are performed to obtain the phase diagram for shapes of a vesicle with a variation in temperature and pressure difference across the membrane. Various interesting vesicle shapes are found, in particular, a series of shape transformations are observed for a vesicle with an initial spherical shape, which changes to a prolate shape, then an oblate shape, and then a stomatocyte shape, with either increasing temperature or decreasing pressure difference across the membrane. (C) 2009 American Institute of Physics.

[doi:10.1063/1.3245307]

Mechanical properties and morphological transformations of solid/fluid membranes have attracted great interest due to their ubiquitous presence in biological systems and frequent appearance in nanosystems. ${ }^{1,2}$ Continuum linear elastic models have been extensively used to study the mechanical properties of both solid and liquid membranes. ${ }^{3-7}$ However, these continuum models may not be able to model systems involving diffusive behavior, thermal fluctuation, and membrane burst and fracture. Hence atomistic simulations have been developed to study the structural and functional behavior of membranes. Since the meso- or macroscopic properties of membranes cannot possibly depend on all the details of the atomic description, coarse-grain atomistic models have been used to study the physical properties of membranes, including formation of membrane structures, ${ }^{8}$ membrane elasticity, ${ }^{8-18}$ thermal fluctuation, ${ }^{8-10,17}$ nanoparticle endocytosis, ${ }^{10,16}$ composition segregation, ${ }^{12,14-16}$ and topological shape changes. ${ }^{18-20}$

A vesicle may change its shape, volume, or surface area, due to the change in properties of its membrane and/or the presence of external loadings. ${ }^{21}$ A phase diagram for vesicle shape transformation at different membrane properties and external loading conditions reveals important thermodynamic behavior of vesicle. So far, the phase diagram for vesicle shape transformation has been only partially explored. For example, phase diagrams at different spontaneous curvatures and vesicle volumes were studied in Ref. 22. Phase diagrams concerning area difference and a change in volume was studied in Ref. 23. Different phases of vesicle shapes, ranging from stomatocytes, pears, prolates, and oblates, were obtained. ${ }^{19,20,23}$ However, the phase diagram for shape transformation of a vesicle involving the variation of temperature and the pressure difference across the membrane is still not available. Here coarse-grained molecular dynamics (CGMD) simulations are performed to obtain the phase diagram for vesicle shape transformation at various temperatures and cross-membrane pressure differences.

\footnotetext{
a) Author to whom correspondence should be addressed. Electronic mail: msezyw@nus.edu.sg. FAX: 6567763604.
}

The vesicle membrane is assumed to consist of singlelayered particles with diameter $a_{0}$, which is also the membrane thickness. ${ }^{17}$ For particle $i, i \in[1, N]$, where $N$ is the total number of particles, five degrees of freedom are considered, that is, $\left(\mathbf{x}_{i}, \mathbf{n}_{i}\right)$, where $\mathbf{x}_{i}$ is the position of particle $i$ and $\mathbf{n}_{i}$ is the surface normal vector at particle $i$, subject to the constraint that $\mathbf{n}_{i} \cdot \mathbf{n}_{i}=1$. The unit vector between particles $i$ and $j$, is $\overline{\mathbf{x}}_{i j}=\mathbf{x}_{i j} / r_{i j}$, where $\mathbf{x}_{i j}=\mathbf{x}_{j}-\mathbf{x}_{i}$, and $r_{i j}=\left|\mathbf{x}_{i j}\right|$. A modified Lenard-Jones (LJ) potential was employed to overcome the drawback of the standard LJ 12-6 potential, which was found to be too short to stabilize a liquid phase. ${ }^{18}$ The modified pair potential energy between particles $i$ and $j$ is of the following form: ${ }^{17}$

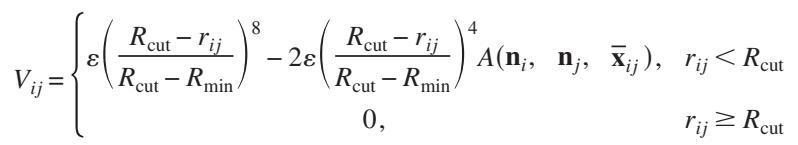

where, $\varepsilon$ is the bonding energy between the two particles, $R_{\text {cut }}=2.5 a_{0}$ is the cutoff distance of the interaction, $R_{\text {min }}$ $=2^{1 / 6} a_{0}$ is introduced so that the modified potential has the same bonding energy and equilibrium position as the standard LJ 12-6 potential; and $A\left(\mathbf{n}_{i}, \mathbf{n}_{j}, \overline{\mathbf{x}}_{i j}\right)$ is the penalty function related to the spontaneous curvature of the membrane surface at the two particle positions. Here it is assumed that the flat membrane is energetically favorable, that is, the spontaneous curvature is zero. Hence if $\mathbf{n}_{i}$ and $\mathbf{n}_{j}$ are parallel, $A\left(\mathbf{n}_{i}, \mathbf{n}_{j}, \overline{\mathbf{x}}_{i j}\right)=1$ and the interaction energy between the two particles is at a minimum; when $\mathbf{n}_{i}$ and $\mathbf{n}_{j}$ are antiparallel, $A\left(\mathbf{n}_{i}, \mathbf{n}_{j}, \overline{\mathbf{x}}_{i j}\right)=-1$ and the interaction energy is at a maximum. Such consideration is consistent with the observation that a lipid molecule in a bilayer membrane is extremely unlikely to change its direction. ${ }^{1}$ Here, $A\left(\mathbf{n}_{i}, \mathbf{n}_{j}, \overline{\mathbf{x}}_{i j}\right)=1+\alpha(B-1)$, where, $\alpha$ is the energy penalty factor for membrane deviating from its flat state, and

$$
B=\mathbf{n}_{i} \cdot \mathbf{n}_{j}-\left(\mathbf{n}_{j} \cdot \overline{\mathbf{x}}_{i j}\right)\left(\mathbf{n}_{i} \cdot \overline{\mathbf{x}}_{i j}\right) .
$$

In the present study, $\alpha=1.5$, since the reference state of the membrane is assumed to be flat, hence its spontaneous curvature is not considered.

The governing equation of motion for particle $i$ can be written as 


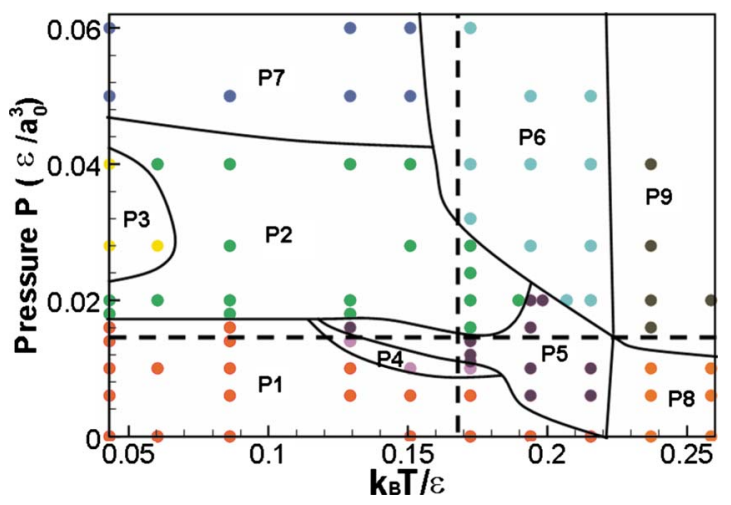

FIG. 1. (Color online) Phase diagram for vesicle shapes as a function of cross-membrane pressure difference and temperature. In total, nine phases (P1-P9) were determined. The dots denote the simulation cases, the horizontal dashed line indicates the phase transitions with increasing temperature at a constant pressure difference, and the vertical dashed line indicates the phase transitions with increasing pressure difference at a constant temperature.

$$
m_{i} \mathbf{x}_{i}=-\frac{\partial V}{\partial \mathbf{x}_{i}}+P \mathbf{n}_{i},
$$

where $P$ is the pressure difference across the membrane and for the surface normal vector, the governing equation concerning evolution is

$$
\tilde{m}_{i} \mathbf{n}_{i}=-\frac{\partial V}{\partial \mathbf{n}_{i}}+\left(\frac{\partial V}{\partial \mathbf{n}_{i}} \cdot \mathbf{n}_{i}\right) \mathbf{n}_{i}-\tilde{m}_{i}\left(\dot{\mathbf{n}}_{i} \cdot \dot{\mathbf{n}}_{i}\right) \mathbf{n}_{i},
$$

where $\tilde{m}_{i}$ is a pseudomass and the right-hand side conforms to the constraint $\mathbf{n}_{i} \cdot \mathbf{n}_{i}=1$. The integration method which was proposed by Beeman ${ }^{24}$ is used. The position update algorithm is,

$$
\mathbf{x}_{n+1}=\mathbf{x}_{n}+\mathbf{v}_{n} \Delta t+\frac{4 \mathbf{x}_{n}-\mathbf{x}_{n-1}}{6}(\Delta t)^{2},
$$

and that for velocity is

$$
\mathbf{v}_{n+1}=\mathbf{v}_{n}+\frac{5 \mathbf{x}_{n+1}+8 \mathbf{x}_{n}-\mathbf{x}_{n-1}}{12} \Delta t .
$$

The following dimensionless scheme is used: for mass $m^{*}$ $=m / m_{0}$, where $m_{0}$ is the mass of the particle, for distance $r^{*}=r / a_{0}$ and for energy $V^{*}=V / \varepsilon$. The derived unit for time is $t_{0}=a_{0} \sqrt{m_{0} / \varepsilon}$ and the velocity is $v_{0}=a_{0} / t_{0}$.

Systematic parametric studies by varying temperature and pressure difference across the membrane have been performed to obtain the phase diagram for vesicle shapes. In all the simulations, each vesicle contains 5072 particles and the initial radius of the vesicle is $19.97 a_{0}$. A positive pressure difference across the membrane implies that the pressure inside the vesicle is lower than that outside the vesicle. The Berendsen method was used to maintain an approximately constant temperature during the simulations.

The predicted phase diagram is shown in Fig. 1. In total, nine phase zones (P1-P9) were observed within a temperature range of $\left[0-0.275 k_{B} T / \varepsilon\right]$, where $k_{B}$ is Boltzmann's constant and the pressure difference range of $\left[0-0.06 \varepsilon / a_{0}^{3}\right]$. The typical shapes for the nine phases are shown in Fig. 2 with the shapes (P1)-(P9) corresponding to the phases $\mathrm{P} 1-\mathrm{P} 9$, respectively. It is seen that various shapes are observed, rang-
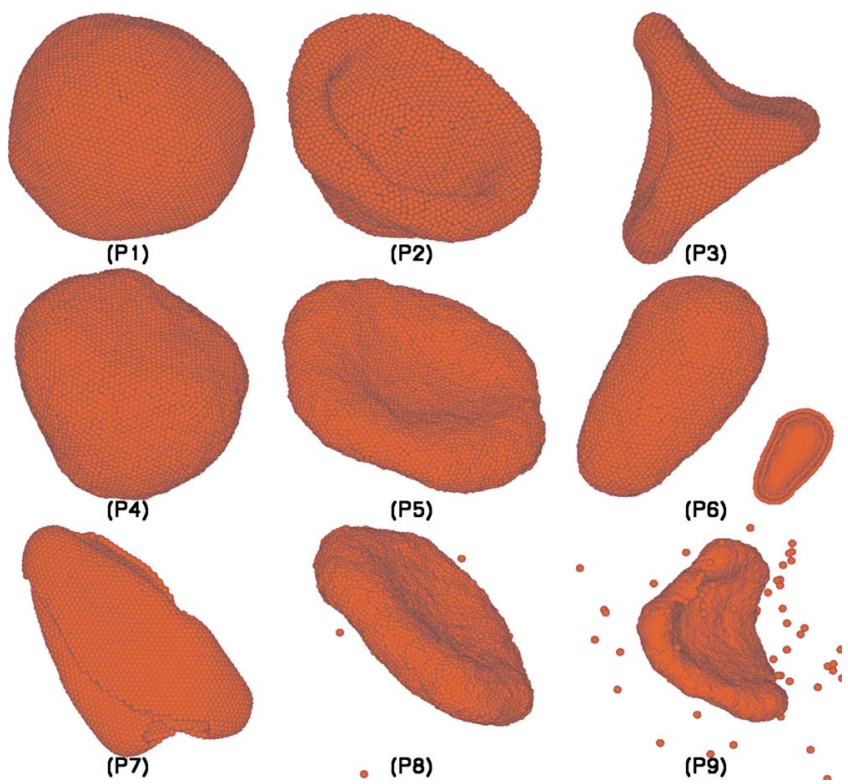

FIG. 2. (Color online) Vesicle shapes as a function of the cross-membrane pressure difference and temperature: the shapes from (P1) to (P9) correspond to the phases from $\mathrm{P} 1$ to $\mathrm{P} 9$, respectively, in Fig. 1. Note that the smaller cross section view in (P6) shows the double-layer structure.

ing from a spherical shape $(\mathrm{P} 1)$, to a stomatocyte shape $(\mathrm{P} 2)$, to a multiridge shape (P3), to a prolate shape $(\mathrm{P} 4)$, to an oblate shape (P5), to a double-layer vesicle (P6), to a compact solidlike shape (P7), to an oblate shape with particle evaporation (P8), and to a stomatocyte shape with particle evaporation (P9). Hence the vesicle shapes are strongly dependent on both temperature and cross-membrane pressure difference.

It was predicted by using a continuum model that a vesicle can change from a spherical shape to a prolate shape and then to an oblate shape, and then to a stomatocyte shape by decreasing vesicle volume. ${ }^{21,22}$ One can see by examining Fig. 1, that the present model also predicts the same series of shape transformations by either increasing the temperature or decreasing the pressure difference. For example, for a pressure difference range of $\left[0.011 \varepsilon / a_{0}^{3}-0.018 \varepsilon / a_{0}^{3}\right]$, the vesicle is able to change from a spherical shape (P1), to a prolate (P4), to an oblate (P5), and then to an evaporated stomatocyte shape (P8), with a gradual increase in temperature (see the horizontal dashed line in Fig. 1). For a temperature range of $\left[0.0115 k T_{B} / \varepsilon-0.0185 k_{B} T / \varepsilon\right]$, a series of shape transformations from a spherical vesicle $(\mathrm{P} 1)$, to a prolate $(\mathrm{P} 4)$, to a oblate (P5), and then to a stomatocyte shape (P2) take place with a decrease in $P$ (see the vertical dashed line in Fig. 1). Hence the effect of temperature increase or a decrease in pressure difference can lead to the same shape transformations as those caused by a change of volume.

The shape transformations induced by pressure difference can be understood in a similar way as that induced by the vesicle volume change predicted by the continuum model. $^{21}$ However, the shape transformations induced by a change in temperature require greater analysis. The transformation of a vesicle from a spherical to a nonspherical shape (for example, a prolate) can be considered as the buckling process of a spherical vesicle under uniformly pressure. The magnitude of the uniform compressive stress in the membrane is $\sigma=P R / 2 a_{0}$, where $P$ is the pressure difference, $R$ is the radius of the vesicle, and $a_{0}$ is the thickness of the 


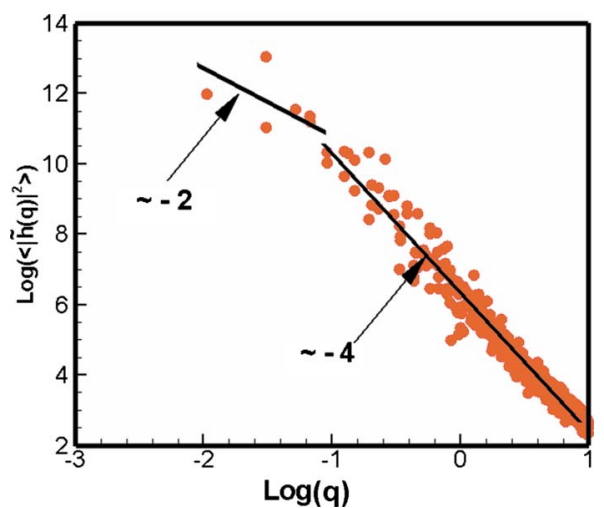

FIG. 3. (Color online) The relation between $\left\langle|\widetilde{h}(q)|^{2}\right\rangle$ vs $q$, where $L$ is the simulation cell size. A linear scaling between $\left\langle|\widetilde{h}(q)|^{2}\right\rangle$ and $q^{-4}$ is evident at a large norm of the wave vector. The thermal fluctuation is performed at $k_{B} T / \varepsilon=0.22$.

vesicle membrane. If the pressure difference increases beyond a certain limit, the spherical form of the vesicle becomes unstable. The critical pressure difference is $P_{c r}$ $=\left[8 K \sqrt{3\left(1-\nu^{2}\right)}\right] /\left(a_{0} R^{2}\right)$, where $K$ is the bending rigidity and $\nu$ is Poisson's ratio of the membrane. ${ }^{25}$ It is seen that an increase in the vesicle radius (or the membrane surface area) or a decrease in bending rigidity can cause an earlier transformation from a spherical to a nonspherical shape.

It is known ${ }^{21}$ that the membrane bending rigidity $K$ is related to the spectrum of thermal fluctuation modes by $\left\langle|\widetilde{h}(q)|^{2}\right\rangle=k_{B} T /\left(\gamma q^{2}+K q^{4}\right)$, where $\widetilde{h}(q)$ is the Fourier transform of thermal fluctuation $h, q$ is the norm of the wave vector $\boldsymbol{q}=\left(q_{x}, q_{y}\right)=2 \pi\left(n_{x}, n_{y}\right) / L, L$ is the lateral size of the solid membrane, $\gamma$ is the membrane tension, and $k_{B} T$ is the thermal energy. If the membrane tension is small or if the norm of the wave vector is large $(\gg 1)$, the membrane tension $\gamma$ can be ignored. Thus one can perform thermal equilibrations and extract the membrane bending rigidity $K$ at different temperatures. The total number of particles $N$ of the membrane in the simulations is 4096. A periodic boundary condition is used in the thermal equilibration. For example, the relation between $\left\langle|\widetilde{h}(q)|^{2}\right\rangle$ versus $q$ is shown in Fig. 3 for a thermal fluctuation performed at $k_{B} T / \varepsilon=0.22$. It can be seen that a linear scaling between $\left\langle|\widetilde{h}(q)|^{2}\right\rangle$ and $q^{-4}$ is evident for large norms of the wave vector. The extracted $K$ value is $1.58 \varepsilon$ or $7.2 k_{B} T$ at a temperature of $k_{B} T / \varepsilon=0.22$, which is comparable to experimentally measured values of biological membranes, $3-30 k_{B} T .^{21}$ The simulation results shown in Fig. 4 show a clear decreasing tendency of the bending rigidity with an increase in temperature. This result is consistent with the first-order perturbation theory ${ }^{4}$ and the experimental observation. ${ }^{26}$ It is also found that this tendency can be well fitted by $K=K_{0} e^{-\eta T}$, where the bending stiffness at $0 \mathrm{~K}, K_{0}$ $=10.1 \varepsilon$, and $\eta=1.12 \times 10^{-5} k_{B} / \varepsilon$.

The CGMD model used here is coarser than that used in previous studies. ${ }^{12,18-20}$ In the present model, a membrane particle with a diameter of membrane thickness and described by five degrees of freedom, actually represents a number of lipid molecules. In addition, this model eliminates the explicit consideration of solvent molecules, and their effect is considered by the effective particle interaction poten-

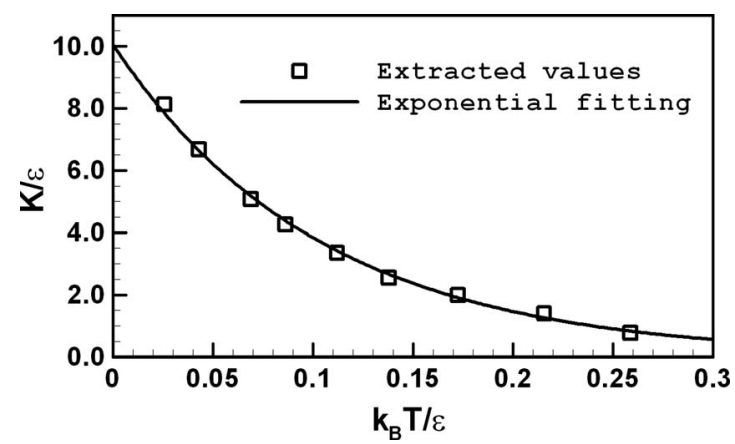

FIG. 4. Variation of membrane bending rigidity with temperature. The extracted values of bending rigidity at different temperatures can be well fitted by the following exponential function: $K=K_{0} e^{-\eta T}$, where $K_{0}=10.1 \varepsilon$ and $\eta=1.12 \times 10^{-5} k_{B} / \varepsilon$.

tial. Furthermore, the existence of the liquid membrane phase is catered for, enabling the modeling of fast particle diffusion. Finally, the most important material quantity, the bending rigidity extracted by using the present model reproduces experimental results.

In summary, systematic CGMD simulations were performed to obtain the phase diagrams for shapes of a vesicle at different cross-membrane pressure differences and temperatures. It was found that the temperature increase or a decrease in pressure difference can lead to a series of shape transitions, different vesicle shapes were observed and possible reasons for the shape transition were also provided.

${ }^{1}$ M. Tanaka and E. Sackmann, Nature (London) 437, 656 (2005).

${ }^{2}$ J. C. Meyer, A. K. Geim, M. I. Katsnelson, K. S. Novoselov, T. J. Booth, and S. Roth, Nature (London) 446, 60 (2007).

${ }^{3}$ Z. C. Tu and Z. C. Ou-Yang, J. Comput. Theor. Nanosci. 5, 1192 (2008).

${ }^{4}$ L. Peliti and S. Leibler, Phys. Rev. Lett. 54, 1690 (1985).

${ }^{5}$ S. T. Milner and S. A. Safran, Phys. Rev. A 36, 4371 (1987).

${ }^{6}$ U. Seifert, Phys. Rev. Lett. 70, 1335 (1993).

${ }^{7}$ F. Julicher and R. Lipowsky, Phys. Rev. Lett. 70, 2964 (1993).

${ }^{8}$ J. M. Drouffe, A. C. Maggs, and S. Leibler, Science 254, 1353 (1991).

${ }^{9}$ H. Noguchi and G. Gompper, Phys. Rev. E 73, 021903 (2006).

${ }^{10}$ H. Noguchi and M. Tanasu, Biophys. J. 83, 299 (2002).

${ }^{11}$ G. Brannigan, Phys. Rev. E 72, 011915 (2005).

${ }^{12}$ I. R. Cooke, K. Kremer, and M. Deserno, Phys. Rev. E 72, 011506 (2005)

${ }^{13}$ J. D. Revalee, M. Laradji, and P. B. S. Kumar, J. Chem. Phys. 128, 035102 (2008).

${ }^{14}$ P. B. S. Kumar, G. Gompper, and R. Lipowsky, Phys. Rev. Lett. 86, 3911 (2001).

${ }^{15}$ M. Laradji and P. B. S. Kumar, J. Chem. Phys. 123, 224902 (2005).

${ }^{16}$ K. A. Smith, D. Jasnow, and A. C. Balazs, J. Chem. Phys. 127, 084703 (2007).

${ }^{17} \mathrm{~J}$. Li, G. Lykotrafitis, S. L. Zhang, and S. Suresh, GEM4 Summer School Lecture Notes, Singapore, 2 July 2007 (unpublished).

${ }^{18}$ M. Deserno, Macromol. Rapid Commun. 30, 752 (2009).

${ }^{19}$ A. J. Markvoort, P. Spijker, A. F. Smeijers, K. Pieterse, R. A. van Santen, and P. A. J. Hilbers, J. Phys. Chem. B 113, 8731 (2009).

${ }^{20}$ A. J. Markvoort, R. A. van Santen, and P. A. J. Hilbers, J. Phys. Chem. B 110, 22780 (2006).

${ }^{21}$ D. Boal, Mechanics of the Cell (Cambridge University Press, Canmbridge, 2002), pp. 158-160.

${ }^{22}$ L. Miao, B. Fourcade, M. Rao, and M. Wortis, Phys. Rev. A 43, 6843 (1991)

${ }^{23}$ L. Miao, U. Seifert, M. Wortis, and H. G. Döbereiner, Phys. Rev. E 49, 5389 (1994).

${ }^{24}$ D. Beeman, J. Comput. Phys. 20, 130 (1976).

${ }^{25}$ E. Schwerin, Z. Angew. Math. Mech. 2, 81 (1922).

${ }^{26}$ J. Pan, S. Tristram-Nagle, N. Kucerka, and J. F. Nagle, Biophys. J. 94, 117 (2008). 